\title{
Determination of the Geometric Form of a Plane of a Tectonic Gap as the Inverse III-posed Prob- lem of Mathematical Physics
}

\author{
Dmitry Sirota ${ }^{1}$, Vadim Ivanov ${ }^{1}$ \\ ${ }^{1}$ T.F. Gorbachev Kuzbass State Technical University, 650000, 28 Vesennyaya St., Kemerovo, Russia
}

\begin{abstract}
Any mining operations influence stability of natural and technogenic massifs are the reason of emergence of the sources of differences of mechanical tension. These sources generate a quasistationary electric field with a Newtonian potential. The paper reviews the method of determining the shape and size of a flat source field with this kind of potential. This common problem meets in many fields of mining: geological exploration mineral resources, ore deposits, control of mining by underground method, determining coal self-heating source, localization of the rock crack's sources and other applied problems of practical physics. This problems are ill-posed and inverse and solved by converting to FredholmUryson integral equation of the first kind. This equation will be solved by A.N. Tikhonov regularization method.
\end{abstract}

\section{Introduction}

The problem of stability of natural and technogenic massifs is bound to definition of geometry of the center of differences of mechanical tension which is a source of a seismic event (SE). According to D. L. Anderson's theory, the sources of SE is the some volume area of rocks surrounding the place of the arising gap and corresponding to a zone of nonreversible deformations and maximal differences of mechanical tension. In this definition gaps are violations of a structure of rocks. D. L. Anderson [1,2] considers that in the massif of rocks there is a plane on which there are a gap and shift of blocks relatively each other. Let's consider that the plane has the form ellipse as at any explosive violation there has to be its attenuation towards the periphery of a gap. Using harbingers of seismic events we can define various characteristics of SE. In this article we shall use the electric harbingers of SE.

The search for electric harbingers of SE (rock cracks, earthquake) has led to the beginning of the nineties of the last century, to the discovery of the mechanism of electrification of pockets the forthcoming SE at the stage of the avalanche - unstable fracturing, i.e. at the final stage of preparation. It was shown both theoretically and experimentally that each crack in the process of the emergence and rapid spread bears at its apex a charge of the same sign, when multiple their accumulation in the focus leads to a strong electrification of rocks and causes severe abnormalities in the electrical field in the atmosphere and on the surface of the earth. The definition of the shape and size of the area of electrification of rocks on measurements on 
the earth's surface can serve as the basis of the forecast power of the impending seismic events and predict the time of occurrence of the event.

The problem of definition of the shape of the area is the inverse ill-posed problems of mathematical physics. We can formulate this problem as Fredholm-Uryson integral equation of the first kind, which will be solved by A. N. Tikhonov regularization method [3-14].

\section{Results and discussions}

\subsection{Formulation and solution of the direct problem}

Let us assume that the natural electric field is generated by flat source arbitrary shape $S_{\mathrm{p}}$ which is inclined at an angle $\gamma \in\left[0^{0} ; 70^{0}\right]$ and located at a certain depth $z_{M}=h$ (Fig 1) or $z_{M}=h+z_{1}$ (Fig 2). We also assume that the sources containing space is homogeneous and isotropic with one (Fig 1) or two plane-parallel borders (Fig 2).
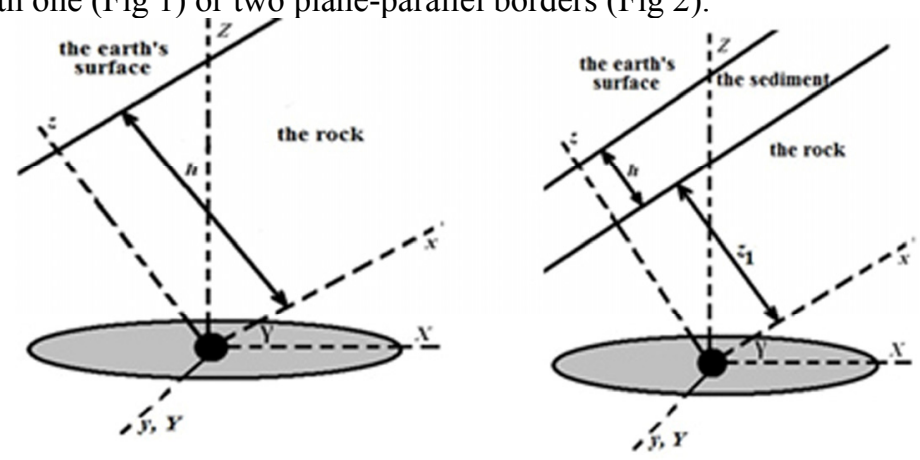

Fig. 1, 2. The schemes of the containing space and flat field source

The value of potential of such a source at an arbitrary measurement point $M$ on the surface will be determined by the general formula [13]

$$
U^{M}=\int_{S_{P}} u^{M} d S_{P},
$$

where $S_{P}$ - flat source of electric field; $u^{M}$ - the potential of point sources that determined by one of the two formulas (Fig 1 or Fig 2)

$$
\begin{aligned}
& u_{1}^{M}=\frac{C}{\left[D+z_{M}^{2}\right]^{1 / 2}} \text { or } \\
& u_{2}^{M}=C \int_{0}^{\infty} \frac{J_{0}(m D) \exp \left(-m z_{M}\right)}{1+W \exp (-2 m h)} d m=C \sum_{k=1}^{11} \frac{q(k)}{\left[D+\left[z_{M}+2(k-1)\right]^{2}\right]^{1 / 2}},
\end{aligned}
$$

where $J_{0}(m D)$ - Bessel function; $W=\left(\rho_{2}-\rho_{1}\right) \cdot\left(\rho_{2}+\rho_{1}\right)^{-1}-$ the reflection coefficient of the second layer; $\rho_{1,2}-$ electrical resistivity layers, $(\mathrm{Om} \cdot \mathrm{m})$ $D=\left(x_{P}-x_{M}\right)^{2}+\left(y_{P}-y_{M}\right)^{2} ; M-$ point of measurement of potential, $P$ - the point located in the flat sources; $q(k)$ - the coefficients of the approximation formula; $C$ - the coefficient, which characterizes the intensity of the field. 
Since the source is located at an angle $\gamma$ to the earth's surface, we will give the formula to calculate the coordinates of measuring points in the new system $O X Y$ $X_{M}=x_{M} \cos \gamma+z_{M} \sin \gamma, Z_{M}=-x_{M} \sin \gamma+z_{M} \cos \gamma$.

For integration in the region $S_{p}$ let us use the polar coordinate system $(\rho, \varphi)$ where $\varphi \in[0 ; 2 \pi], r \in[0 ; \rho(\varphi)]$ and $X_{P}=r \cos \varphi, Y_{P}=r \sin \varphi$.

Additionally, let us turn to dimensionless quantities with formula $w \cdot h^{-1}=\bar{w}$, where $w$ are all linear parameter with dimension $[\mathrm{m}]$. Further, for simplicity, we will not write a line above the letters.

Thus, formula (1) will converts to $U^{M}=\int_{0}^{2 \pi} d \varphi \int_{0}^{\rho(\varphi)} u^{M}(r, \varphi) d r$ and with (2.1) and (2.2) then converts to

$$
U_{1}^{M}=C \cdot h \cdot \int_{0}^{2 \pi} d \varphi \int_{0}^{\rho(\varphi)} \frac{r d r}{\sqrt{R}} \quad \text { or } \quad U_{2}^{M}=C \cdot h \cdot \int_{0}^{2 \pi}\left[\sum_{k=1}^{11} q(k) \int_{0}^{\rho(\varphi)} \frac{r d r}{\sqrt{R}}\right] d \varphi,
$$

where $R=r^{2}+B \cdot r+A, \quad B=-2\left(X_{M} \cos \varphi+Y_{M} \sin \varphi\right), A=X_{M}^{2}+Y_{M}^{2}+Z_{M}^{2}$ for (Fig 1) and $A=X_{M}^{2}+Y_{M}^{2}+\left[Z_{M}+2(k-1)\right]^{2}$ for (Fig 2). The inner integral in (3) we can calculate analytically: $\int_{0}^{\rho(\varphi)} \frac{r d r}{\sqrt{R}}=\left.\left(\sqrt{R}-\frac{B}{2} \ln \left|\bar{r}+\frac{B}{2}+\sqrt{R}\right|\right)\right|_{0} ^{\rho(\varphi)}$

Thus we have the Fredholm-Urysohn integral equation (IE) [3, 4] of the 1-st kind with unknown parameter $\rho(\varphi)$ to determine the shape of the source field with the Newtonian potential

$$
\int_{0}^{2 \pi} K(M, \rho(\varphi)) d \varphi=U^{*}(M)
$$

where $K$ - nonlinear kernel of equation; $U^{*}$ - values of potential measured on the earth's surface in the point $M$.

\subsection{Solution of the inverse problem}

The inverse problem is to determinate the function $\rho(\varphi)$ if we know potential value on the earth's surface. For the definition this function we consider the problem of finding the minimum of regularized functional of A.N. Tikhonov

$$
\Phi(\rho)=\mathrm{T}\left(x_{M}, y_{M}, \rho(\varphi)\right)+\alpha \Omega(\rho(\varphi))
$$

where $\mathrm{T}$ - a measure of the error of measurement of the value of the potential; $\Omega=\int_{0}^{2 \pi}\left(\rho(\varphi)^{2}+\left(\frac{d \rho(\varphi)}{d \varphi}\right)^{2}\right) d \varphi$ - the stabilizing functional; $\alpha$ - the regularization parameter.

In article [15] we assumed, that the measure of potential value is on a one-dimensional region ( $y_{M}=0$ for example) therefore, as the measure has been used the integral 
$\mathrm{T}(\rho)=\int_{a}^{b}\left[A\left(x_{M}, \rho(\varphi)\right)-U^{*}\left(x_{M}\right)\right]^{2} d x_{M}$ where $A\left(x_{M}, \rho(\varphi)\right.$ - non-linear integral direct

modeling operator (3); $U^{*}$ - the right part of the IE (4), which in practice is set experimentally, and for test problems is determined by solving a direct problem using formulas (3) with the addition of a random correction simulating inaccuracies in full-scale measurements.

In this article we will assume, that the measure of potential value will be on a twodimensional region therefore, as the measure has been used the sum $\mathrm{T}(\rho)=\sum_{k=1}^{N}\left[A\left(M_{k}, \varphi, \rho(\varphi)\right)-U^{*}\left(M_{k}\right)\right]^{2}$ where $N$ is the number of measurement points $M$.

We shall seek the minimum of the functional (5) by the method of conjugate gradients (CG) [16], whose general iterative scheme has the form

$$
\rho_{q+1}=\rho_{q}+k_{q} \cdot I_{q}
$$

where $I_{q}=-\frac{d \Phi\left(\rho_{q}\right)}{d \rho}+\gamma_{q} \cdot I_{q-1}, q-$ iteration index; $k_{q}-$ minimization step; $\gamma_{q}-$ CGcoefficient; $I_{0}=-\frac{d \Phi\left(\rho_{0}\right)}{d \rho}$ where $\frac{d \Phi\left(\rho_{q}\right)}{d \rho}=\Phi_{\rho}^{\prime}-$ the Freshet derivative of the functional (3); .

As well as know from the functional analysis the Freshet derivative of functionals $\mathrm{T}$ and $\Omega$ will be calculated by the following formulas

$$
\begin{gathered}
\frac{d \mathrm{~T}(\rho)}{d \rho}=2 \sum_{k=1}^{N}\left[\left[A\left(M_{k}, \varphi, \rho(\varphi)\right)-U^{*}\left(M_{k}\right)\right] \times\left.\left(\frac{d K\left(M_{k}, \varphi, \rho(\varphi)\right)}{d \rho}\right)\right|_{\varphi=t}\right] \\
\frac{d \Omega}{d \rho}=\left.2\left(\rho(\varphi)-\frac{d^{2} \rho(\varphi)}{d \varphi^{2}}\right)\right|_{\varphi=t},
\end{gathered}
$$

where $\frac{d K\left(M_{k}, \varphi, \rho(\varphi)\right)}{d \rho}=\frac{\rho(\varphi)}{\sqrt{R_{\varphi}}}$ for Fig 1 and $\frac{d K\left(M_{k}, \varphi, \rho(\varphi)\right)}{d \rho}=\sum_{k=1}^{11} \frac{q(k) \cdot \rho(\varphi)}{\sqrt{R_{\varphi}}}$ for Fig 2, $R_{\varphi}=\rho^{2}(\varphi)+B \cdot \rho(\varphi)+A t-$ a fixed value of the argument $\varphi$, which defines the variable of differentiation $\rho(t)$.

\section{Numerical realization}

Numerical realization the above formulas consists of the following aspects.

The first is the numerical integration and differentiation functions in (5). For this we shall define the grid to the variable $\varphi$ with step $u=\frac{2 \pi}{N}, N-$ the number of nodes on the grid, at each point where we shall compute $\rho(\varphi)$. To calculate the integral in (4), we shall use the Simpson formula. To calculate the second order derivative in (7), we shall use cen- 
tral deference relation $\frac{d^{2} \rho_{k}}{d \varphi^{2}}=\frac{\rho_{k-1}-2 \rho_{k}+\rho_{k+1}}{u^{2}}, \frac{d^{2} \rho_{1}}{d \varphi^{2}}=\frac{\rho_{N-1}-2 \rho_{1}+\rho_{2}}{u^{2}}$, $\frac{d^{2} \rho_{1}}{d \varphi^{2}}=\frac{d^{2} \rho_{N}}{d \varphi^{2}}$

The second is to calculate three coefficients: $k_{q}, \gamma_{q}, \alpha$. To find the step of minimizing $k_{q}$, we will use the "golden section" method for the objective function $\Phi(\rho+k \cdot I)$. Numerical experiments have shown that this function is unimodal on the interval $k \geq 0$. Since it is impossible in advance to determine the interval at which the minimum point is contained, we shall adhere to the algorithm described in [15]. To calculate the CG-coefficient $\gamma_{q}$ we can use one of the multiple existing formulas [16]. In this article we shall use

$$
\gamma_{q}=\max \left\{0 ; \min \left\{\frac{\Phi_{q+1}^{\prime} \bullet \Phi_{q+1}^{\prime}}{\Phi_{q}^{\prime} \bullet \Phi_{q}^{\prime}} ; \frac{\Phi_{q+1}^{\prime} \bullet\left(\Phi_{q+1}^{\prime}-\Phi_{q}^{\prime}\right)}{\Phi_{q}^{\prime} \bullet \Phi_{q}^{\prime}}\right\}\right\}
$$

where $a \bullet b$ is the dot product [14]. To calculate coefficient $\alpha$ we shall use the iterative method [17] which is expressed in the formula $\alpha_{q}=\alpha_{0}(1+q)^{-0,25}$ where start value $\alpha_{0}=10^{-4}[17]$.

The third is the test program. Let us consider a test region in the form ellipse the dimensionless equation of which in the polar coordinate system has the form

$$
\rho(\varphi)=\frac{a b}{h \sqrt{a^{2} \sin ^{2} \varphi+b^{2} \cos ^{2} \varphi}},
$$

where $a=50, b=15, h=150$ for Fig. 1 and $h=25$ meters for Fig. 2. The start region for (6) is the circle with the radius $40 \mathrm{~m}$. To simulate errors in full-scale measurements, we will add a random correction to the potential value $U^{*}=U^{e x}+\beta \cdot($ rand $-0,5)$, where rand are uniformly distributed numbers on the interval $[0 ; 1], U^{\mathrm{ex}}-$ the exact calculated value of the potential according to the formula (3), $\beta=\{0,05 ; 0,5\}$.

We will show in Fig. 3, 4 the graphic result of restoration the shape of flat sources for Fig. 1 and Fig. 2.

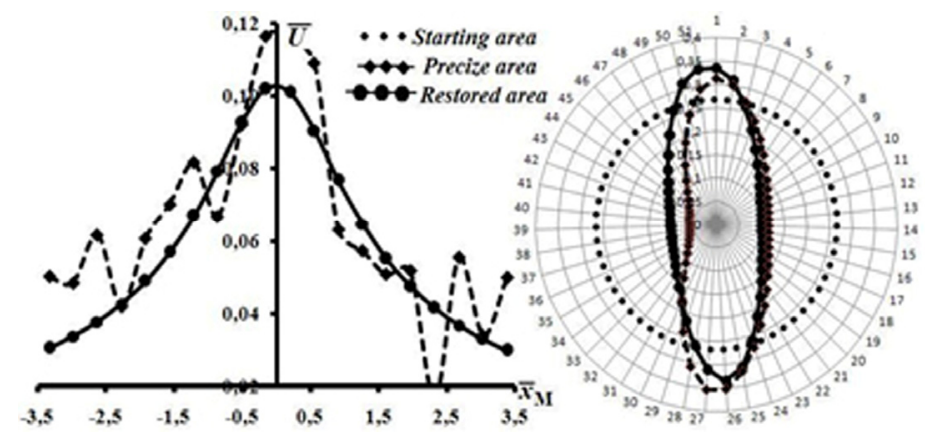

Fig. 3. Restoration of the area and potential value after 100 iterations CG-methods and $\beta=0,05$. 

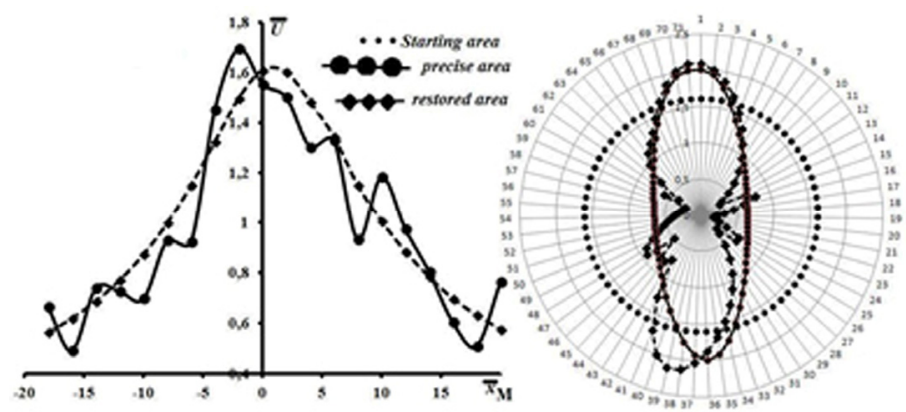

Fig. 4. Restoration of the area and potential value after 150 iterations CG-methods.

\section{Conclusion}

Let's give practical recommendations on the using of the developed methodology some aspects of which was introduced in article [15]. A coordinate grid is constructed on the surface with a step of about 25-50 meters. As we said above these values are used as the right part of IE (4). Then we use the developed program, which removed the random additive.

\section{References}

1. D.L. Anderson, New Theory of the Earth, (London, CUP, 2007)

2. D.L. Anderson, Theory of the Earth, (Hampshire, BSP, 1989)

3. A.N. Tikhonov, V.B. Glasko, J. Comp. Math. Math. Phys., 4, 564, (1964)

4. A.N. Tikhonov, V.B. Glasko, J. Comp. Math. Math. Phys., 5, 463, (1965)

5. J.P. Butler, J.A. Reeds, S.E. Dawson, J. Numer. Anal., 18, 546 (1981)

6. V.A. Morozov, Regularization methods fo ill-posed problems (New York, CRC Press, 1993)

7. F.D.M. Neto, A.J.S. Neto, An introduction to inverse problems with application (New York, Springer, 2013)

8. H.W. Engl, M. Hanke, A. Neubauer, Regularization of inverse problems (London, CUP, 1996)

9. C.R. Vogel, Computational methods for inverse problems (Hamburg, SIAM, 2002)

10. Y. Wang, A.G. Yagola, C. Yang, Optimization and regularization for computational inverse problems and applications (London, Springer, 2011)

11. R.C. Aster, B. Borchers, C.H. Thurber, Parameter estimation and inverse problems (Cambridge, Elsevier Academic Press, 2005)

12. G. Chavent, Nonlinear least squares for inverse problems (New York, Springer, 2009)

13. M. S. Zhdanov, Geophysics, 76, F77 (2011)

14. R. Kress, Inverse problems, 21, 1207 (2005)

15. D. Sirota, E3S Web Conf, 151012 (2017)

16. W.W. Hager, Pacific J. Optim. 2, 35, (2006)

17. A.B. Bakushinsky, M.Yu. Kokurin, A. Smirnova, Iterative methods for ill-posed problems (Berlin/New York, Walter de Gruyter GmbH \& Co. KG2011) 